Fetal Diagnosis and Therapy

\title{
Predictors of Cranial Ultrasound Abnormalities in Intrauterine Growth-Restricted Fetuses Born between 28 and 34 Weeks of Gestation: A Prospective Cohort Study
}

\author{
Soghra Khazardoost ${ }^{a}$ Fahimeh Ghotbizadeh ${ }^{a}$ Behrokh Sahebdel $^{b}$ \\ Fatemeh Nasiri Amiric Masoumeh Shafaat ${ }^{\mathrm{d}}$ Zahra Akbarian-Rad ${ }^{\mathrm{e}}$ \\ Zeinab Pahlavan ${ }^{b}$ \\ a Department of Perinatology, Tehran University of Medical Sciences, Maternal Fetal Neonatal Research Center, \\ Imam Khomeini Hospital, Tehran, Iran; ${ }^{b}$ Department of Perinatology, Tehran University of Medical Sciences, Emam \\ Khomeini Medical Complex, Vali-Asr Hospital, Tehran, Iran; ' Department of Midwifery, Fatemeh Zahra Infertility and \\ Reproductive Health Research Center, Babol University of Medical Sciences, Babol, Iran; ${ }^{\mathrm{d}}$ Department of Obstetrics \\ and Gynecology, Tehran University of Medical Sciences, Emam Khomeini Medical Complex, Vali-Asr Hospital,

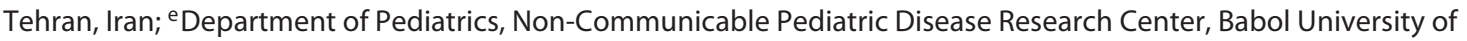 \\ Medical Sciences, Babol, Iran
}

\section{Keywords}

Doppler parameters · Neonatal brain abnormality ·

Intrauterine growth restriction

\begin{abstract}
Background: Doppler parameters have been commonly used for the prediction of neonatal outcomes. However, controversies exist with regard to the value of Doppler parameters in predicting the risk of neurological outcomes among neonates. Objective: This prospective cohort study attempted to assess the value of Doppler parameters in predicting cranial ultrasound abnormalities (CUAs) in intrauterine growth restriction (IUGR) among fetuses at 28-34 weeks of gestation. Methods: This was a prospective cohort study of 83 delivered IUGR fetuses and 75 control fetuses matched for gestational age (GA). The value of mentioned Doppler parameters and GA in predicting the risk of CUAs, including periventricular leukomalacia (PVL), intraventricular hemor-
\end{abstract}

\section{KARGER}

(c) 2018 S. Karger AG, Basel

E-Mail karger@karger.com

www.karger.com/fdt rhage (IVH), and basal ganglia lesions (BGLs), was analyzed. Results: The incidence of CUAs among IUGR fetuses (66.3\%) was significantly higher $(p<0.001)$ than in the control group (40\%). The incidence of neonatal mortality among IUGR fetuses was significantly higher $(p<0.001)$ than in the control group. Absent or reversed end-diastolic velocity (AREDV) in the umbilical artery (UA) and the ductus venosus (DV) after adjustment for GA was associated with increased odds of IVH, PVL, BGLs, and any CUA. Conclusions: GA at birth and AREDV in the UA and the DV within 1 week before childbirth were reliable predictors of CUAs during the neonatal period.

(c) 2018 S. Karger AG, Basel

\section{Introduction}

Intrauterine growth restriction (IUGR) is defined as fetuses with an estimated fetal weight $<10$ centiles and abnormal umbilical artery (UA) Doppler $>95$ centiles $[1$, 
2]. IUGR is associated with a higher risk of neonatal mortality, necrotizing enterocolitis, respiratory distress syndrome, and neurological problems, including neonatal brain abnormality (NBA) [3]. Cranial ultrasound abnormalities (CUAs) such as periventricular leukomalacia (PVL), intraventricular hemorrhage (IVH), basal ganglia lesions (BGLs), and transient periventricular echodensities (TPE) are associated with IUGR $[1,4,5]$. NBAs and CUAs are associated with weak memory, learning dysfunction, and neurological visual impairments [6]. In addition, children with IUGR suffer from lower measures of immediate and long-term memory and functional memory compared with normal children $[7,8]$. Therefore, identifying the predictors of CUAs and NBAs is very important for the management of high-risk pregnant women with IUGR fetuses.

According to the results of previous studies on fetuses with IUGR, neurological outcomes are associated with gestational age (GA) at childbirth [8-10]. There are some clinical standards for fetal surveillance, including the assessment of UA and ductus venosus (DV) and absent or reversed end-diastolic velocity (AREDV). Nowadays, different Doppler parameters are suggested for the prediction of neonatal outcomes. However, there is some controversy about the value of Doppler parameters in accurately predicting the risk of adverse neurological outcomes [9-12]. While the values of fetal Doppler parameters were shown to predict NBAs in one study [13], insufficient evidence is available to determine the value of these parameters for predicting neurological outcomes. The aim of this study was to assess the value of Doppler parameters in predicting neurological outcomes among fetuses at 2834 weeks of gestation.

\section{Methods}

\section{Sample and Setting}

This prospective cohort study was conducted from November 2015 to September 2016 in three educational hospitals affiliated with Tehran University of Medical Sciences, Iran. This study included singleton IUGR and normal fetuses. The exclusion criteria for selecting IUGR fetuses were congenital malformations, genetic abnormalities, and birth weight $\geq 10$ centiles. The estimated fetal weight in fetuses with IUGR was $<10$ centiles and abnormal UA Doppler $>95$ centiles [2]. These fetuses were born between 28 and 34 weeks of gestation.

The eligibility criteria for normal fetuses were the estimated fetal weight between 10 to 90 centiles and lack of congenital malformation and genetic abnormalities [14]. The normal fetuses (control group) were matched for GA at delivery.

The basic characteristics of the samples were gathered using a predesigned questionnaire containing questions about age, body mass index, gravidity, parity, history of abortion, type of childbirth, history of smoking, drug use, and alcohol consumption. In addition, clinical signs and symptoms were recorded among pregnant women such as bleeding, preeclampsia, chronic hypertension, diabetes, hypothyroidism, hyperthyroidism, lupus, heart diseases, asthma, and convulsion.

\section{Procedure}

The GA of all fetuses was assessed using the last menstrual period and ultrasound (US) examination based on the measurement of crown-rump length in the first trimester of pregnancy. The perinatal US examinations were conducted using the USA Siemens ACUSON ANTARES ${ }^{\circledR}$ US machine.

For all fetuses with IUGR, Doppler sonography was performed every $48-72 \mathrm{~h}$ by an experienced operator (Z.P.). The first sonography in fetuses with IUGR was performed under the following conditions: (1) after the diagnosis of IUGR and hospitalization, (2) before the start of betamethasone or $48 \mathrm{~h}$ after the last dose of betamethasone for the purpose of removing the probable effects of drugs on Doppler parameters, (3) if the fetus was not moving, and (4) if the mother had voluntary breathing.

The Doppler parameters were measured using at least three consecutive waveforms with the angle of insonation near zero. A high-pass wall filter of $70 \mathrm{~Hz}$ was used to register low-flow velocities to avoid probable biases. The measured mechanical and thermal indices were as follows:

(1) UA pulsatility index (PI) and resistance index (RI) were measured using the free-floating cord loop. The DV was measured in the midsagittal or transverse section of the fetal abdomen, which was located in the Doppler gate at the DV as the vessel connecting the umbilical vein to the inferior vena cava. A color Doppler demonstration for aliasing due to high velocities was added to ensure correct identification. The sample volume was adjusted to the vessel size, and the angle of isolation was kept as close to zero as possible and never exceeded $30^{\circ}$.

(2) PI and RI for the middle cerebral artery and the posterior cerebral artery were measured using a transverse view of the fetal head at the level of origin from the circle of Willis. Maternal uterine artery PI was obtained transabdominally from the lower lateral quadrant of the abdomen. Two Doppler parameters to alter the brain flow velocity were middle cerebral artery $\mathrm{PI}<5$ th percentile for GA and the cerebroplacental ratio, which was calculated by dividing the middle cerebral artery PI by the UA PI.

(3) The DV as the quantitative Doppler parameter was dichotomized into present or absent/reversed end-diastolic velocity according to presence or absence of diastolic blood flow. AREDV in UA was defined as the absence of antegrade flow in $>50 \%$ of the cycle and persistent in at least two examinations within a 12-h interval. Moreover, AREDV in the DV was defined as absence of antegrade flow during atrial contraction.

Parameters measured within 1 week before childbirth only were considered for data analysis. In cases of repeated measurements, the best measurement was selected for data analysis.

Betamethasone was given in all cases for lung maturation of the fetuses. Preeclampsia was managed based on the available guidelines [15]. Childbirth was considered if the following criteria were present: deceleration cardiotocography, reversed end-diastolic velocity in the UA, AREDV in the DV, or persistent abnormal biophysical profile and maternal complications secondary to preeclampsia. 
The neonatal intracranial US examination was performed sequentially on days 3,7 , and 15 as well as at 40 weeks of corrected postnatal age. Information and sonographic findings related to the neonate period were taken from the report made on the day of the US evaluation by one operator working at the center who was a specialist in radiology and US.

The intracranial US examination included five sagittal and six coronal plane images taken from the anterior fontanel. Any abnormally increased echogenicity of the white matter was reported if the affected region was almost as bright as the choroid plexus according to van Wezel-Meijler et al. [16]. These echodensities were classified as TPE if they were present at $72 \mathrm{~h}$ but disappeared by day 14 [4]. The diagnosis of PVL was based on US examination on day 14 after delivery, and it was scored according to the classification described by de Vries et al. [15]. IVH was classified according to the criteria described by Volpe [17]. BGL was diagnosed if an echodensity or an echolucency was seen at any time within the basal ganglia area [13].

The presence of IVH, PVL, or germinal matrix hemorrhage at any US examination was defined as CUA. On the other hand, those that were present at 40 weeks of corrected postnatal age were defined as late CUAs.

Birth weight values were converted into centiles. The intrapartum data consisted of whether the labor was induced or spontaneous and the presence or absence of meconium-stained liquor. Abnormalities of cardiotocography were classified based on the available guideline [18] using epidural analgesia for labor and mode of childbirth. Cases with elective cesarean childbirth were excluded from the data analysis of intrapartum fetal compromises.

Retinopathy of prematurity was defined the classification of retinopathy of prematurity [19]. Presence of persistent ductus arteriosus required confirmation by echocardiography. Sepsis was defined as a positive result of blood culture in conjunction with clinical signs of systemic infection [20]. Necrotizing enterocolitis was defined and was treated for either proven or suspected cases.

\section{Data Analysis}

Categorical and continuous variables were reported as frequency, percentage, mean, and standard deviation. The $\chi^{2}$ test and the $t$ test were used to compare categorical and continuous variables among IUGR and control fetuses, respectively. Moreover, the logistic regression model was used to evaluate the association of GA and Doppler parameters using NBAs among the newborns. The results were reported with a $95 \%$ confidence interval (CI). Stata v.12 (Stata Corp., College Station, TX, USA) was used for data analysis.

\section{Results}

In this prospective cohort study, 83 pregnant women with IUGR and 75 pregnant women with normal fetuses were recruited. The mean ages of the pregnant women with IUGR and the normal women were $30.03 \pm 4.5$ and $28.6 \pm 5.4$ years, respectively, with no statistically significant difference $(p=0.083)$. No statistically significant difference in the proportion of male and female fetuses in
Table 1. Basic characteristics of the participants $(n=158)$

\begin{tabular}{|c|c|c|c|}
\hline Variables & $\begin{array}{l}\text { Controls } \\
(n=75)\end{array}$ & $\begin{array}{l}\text { IUGR } \\
(n=83)\end{array}$ & $\begin{array}{l}p \\
\text { value }\end{array}$ \\
\hline \multicolumn{4}{|l|}{ Sex } \\
\hline Female & $25(33.3 \%)$ & $32(38.6 \%)$ & \multirow[t]{2}{*}{0.495} \\
\hline Male & $50(66.7 \%)$ & $51(61.5 \%)$ & \\
\hline \multicolumn{4}{|l|}{ Nationality } \\
\hline Non-Iranian & $1(1.3 \%)$ & $4(4.9 \%)$ & \multirow[t]{2}{*}{0.206} \\
\hline Iranian & $74(98.7 \%)$ & $79(95.1 \%)$ & \\
\hline Mother's age, years & $28.6 \pm 5.4$ & $30.0 \pm 4.5$ & 0.080 \\
\hline GA, weeks & $32.1 \pm 1.4$ & $31.6 \pm 2.0$ & 0.083 \\
\hline Smoking & $2(2.7 \%)$ & $1(1.4 \%)$ & 0.567 \\
\hline Body mass index & $28.4 \pm 4.1$ & $30.3 \pm 4.1$ & 0.005 \\
\hline \multicolumn{4}{|l|}{ Gravidity } \\
\hline 1 & $33(44.0 \%)$ & $12(14.5 \%)$ & \multirow[t]{4}{*}{$<0.001$} \\
\hline 2 & $39(52.0 \%)$ & $12(14.5 \%)$ & \\
\hline 3 & $3(4.0 \%)$ & $40(48.2 \%)$ & \\
\hline$\geq 4$ & 0 & $19(22.9 \%)$ & \\
\hline \multicolumn{4}{|l|}{ Parity } \\
\hline 0 & $46(61.3 \%)$ & $22(26.5 \%)$ & \multirow[t]{4}{*}{$<0.001$} \\
\hline 1 & $28(37.3 \%)$ & $29(34.9 \%)$ & \\
\hline 2 & $1(1.3 \%)$ & $17(20.5 \%)$ & \\
\hline 3 & 0 & $15(18.1 \%)$ & \\
\hline \multicolumn{4}{|c|}{ Type of previous deliveries } \\
\hline Cesarean & $5(17.2 \%)$ & $57(93.4 \%)$ & \multirow[t]{2}{*}{$<0.001$} \\
\hline Normal vaginal & $24(82.8 \%)$ & $4(6.6 \%)$ & \\
\hline
\end{tabular}

Values are presented as $n(\%)$ or mean \pm standard deviation. GA, gestational age; IUGR, intrauterine growth restriction.

the IUGR and normal women was reported either. However, statistically significant differences among maternal body mass index, gravidity, parity, GA, and method of previous childbirth were reported between the IUGR and normal women (Table 1). The clinical characteristics of the pregnant women and their fetuses are summarized in Table 2.

The proportion of preeclampsia, diabetes, heart diseases, and asthma among the women with IUGR was significantly higher than in the control group. The proportion of necrotizing enterocolitis among IUGR fetuses was significantly higher than that in the control group. The mean Apgar 1 and 5 scores in the IUGR group were significantly lower than those in the control group (Table 2).

The total incidence of IVH, PVL, and BGLs in IUGR fetuses was significantly higher than that in the control group. The grades of IVH are shown in Table 3. The incidence of CUAs among IUGR fetuses (66.3\%) was significantly higher $(p<0.001)$ than that in the control group $(40 \%)$. In addition, 10 cases $(14.3 \%)$ of late CUAs were 
Table 2. Clinical characteristics of mothers according to the status of their fetuses $(n=158)$

\begin{tabular}{|c|c|c|c|}
\hline Variables & Controls $(n=75)$ & IUGR $(n=83)$ & $p$ value \\
\hline Preeclampsia & $21(28.0 \%)$ & $36(43.4 \%)$ & 0.044 \\
\hline Chronic hypertension & $1(1.3 \%)$ & $3(3.6 \%)$ & 0.362 \\
\hline Diabetes & $11(14.7 \%)$ & $27(32.5 \%)$ & 0.009 \\
\hline Lupus & $5(6.7 \%)$ & $3(3.6 \%)$ & 0.382 \\
\hline Hypothyroidism & $11(14.7 \%)$ & $12(14.5 \%)$ & 0.970 \\
\hline Heart disease & $1(1.3 \%)$ & $9(10.8 \%)$ & 0.014 \\
\hline Asthma & $10(13.3 \%)$ & 0 & 0.001 \\
\hline Convulsion & 0 & $1(1.2 \%)$ & 0.340 \\
\hline Birth weight, $g$ & $1,822.5 \pm 329.1$ & $1,202.3 \pm 426.2$ & $<0.001$ \\
\hline Neonatal intensive care unit & $71(94.7 \%)$ & $83(100 \%)$ & 0.033 \\
\hline Duration of hospitalization, days & $13.4 \pm 6.1$ & $24.4 \pm 12.9$ & $<0.001$ \\
\hline Meconium & $3(4.0 \%)$ & $6(7.2 \%)$ & 0.382 \\
\hline Sepsis & $13(17.3 \%)$ & $25(30.1 \%)$ & 0.060 \\
\hline $\mathrm{pH}$ & $7.3 \pm 0.09$ & $7.2 \pm 0.13$ & 0.029 \\
\hline Necrotizing enterocolitis & 0 & $10(12.1 \%)$ & 0.002 \\
\hline Apgar 1 & $7.5 \pm 1.3$ & $6.5 \pm 1.8$ & $<0.001$ \\
\hline Apgar 5 & $9.1 \pm 0.73$ & $8.3 \pm 0.55$ & $<0.001$ \\
\hline
\end{tabular}

Values are presented as $n(\%)$ or mean \pm standard deviation. IUGR, intrauterine growth restriction.

diagnosed among IUGR fetuses compared to the diagnosis of only 1 case (1.4\%) among control fetuses. No statistically significant difference in the incidence rates of TPE, patent ductus arteriosus, and retinopathy of prematurity between IUGR and control fetuses was reported. The incidence of neonatal mortality among IUGR fetuses was significantly higher $(p<0.001)$ than that in the control group (Table 3 ).

The frequencies of IVH among fetuses with normal, absent, and reversed DV were $16.79,55.56$, and $92.86 \%$, respectively. The frequencies of other abnormalities among fetuses with absent and reversed DV were higher than those in control fetuses (Fig. 1a). In addition, the frequency of abnormalities among fetuses with a present diastolic flow of the UA was considerably lower than that of fetuses with an absent and reversed diastolic flow of the UA (Fig. 1b).

The associations between IUGR, GA, and Doppler parameters are shown in Table 4. IUGR adjusted for GA was associated with a higher risk of IVH, PVL, BGLs, and any CUA. An increase in GA was associated with a significantly lower risk of all cranial abnormalities. An increase in posterior cerebral artery peak systolic velocity after adjustment for GA was associated with a statistically significant lower risk of IVH (odds ratio $[\mathrm{OR}]=0.88,95 \%$ CI 0.79-99), BGLs (OR $=0.85,95 \%$ CI 0.77-0.94), and any CUA $(\mathrm{OR}=0.88,95 \% \mathrm{CI} 0.80-0.96)$. Its association with PVL was not statistically significant.
AREDV in the UA and the DV after adjustment for GA were associated with increased odds of IVH, PVL, BGLs, and any CUA. The adjusted ORs of AREDV in the UA for IVH, PVL, BGLs, and any CUA were 13.61 (95\% CI 5.07-36.59), 4.34 (95\% CI 1.48-12.70), 4.81 (95\% CI 2.13-10.84), and 7.58 (95\% CI 2.46-23.37), respectively. The adjusted ORs of AREDV in the DV for IVH, PVL, BGLs, and any CUA were 7.45 (95\% CI 3.55-15.64), 4.01 (95\% CI 1.49-10.84), 6.66 (95\% CI 2.67-16.59), and 14.46 (95\% CI 3.50-59.75), respectively.

\section{Discussion}

In this prospective cohort study, the value of Doppler parameters in predicting CUAs was assessed. The risk of IVH, PVL, BGLs, and any CUA after adjustment for GA was significantly higher among IUGR fetuses than normal ones. AREDV in the UA and the DV after adjustment for GA was a strong predictor of neonatal CUAs. According to this study, GA as an independent variable was a strong predictor of IVH, PVL, BGLs, and any CUA, indicating that a 1-week increase in GA was accompanied by a significant decrease in the risk of the mentioned abnormalities. Regardless of IUGR, lower GA was an important predictor of NBAs, which was in line with the results of previous studies $[13,21]$. 
Table 3. Incidence of CUAs among IUGR and normal controls fetuses $(n=158)$

\begin{tabular}{|c|c|c|c|}
\hline CUAs & $\begin{array}{l}\text { Controls } \\
(n=75)\end{array}$ & $\begin{array}{l}\text { IUGR } \\
(n=83)\end{array}$ & $p$ value \\
\hline \multicolumn{4}{|l|}{ Intraventricular hemorrhage } \\
\hline Third day (total) & $14(18.7 \%)$ & $30(36.1 \%)$ & 0.014 \\
\hline Grade 1 & $4(28.6 \%)$ & $20(66.7 \%)$ & $<0.001$ \\
\hline Grade 2 & $4(28.6 \%)$ & $10(33.3 \%)$ & \\
\hline Grade 3 & $6(42.9 \%)$ & 0 & \\
\hline Grade 4 & 0 & 0 & \\
\hline Seventh day (total) & $12(16.0 \%)$ & $24(31.6 \%)$ & 0.025 \\
\hline Grade 1 & $11(91.7 \%)$ & $15(62.5 \%)$ & 0.254 \\
\hline Grade 2 & 0 & $5(20.8 \%)$ & \\
\hline Grade 3 & $1(8.3 \%)$ & $3(12.5 \%)$ & \\
\hline Grade 4 & 0 & $1(4.2 \%)$ & \\
\hline Fifteenth day (total) & $14(18.7 \%)$ & $7(8.4 \%)$ & 0.443 \\
\hline Grade 1 & $9(64.3 \%)$ & $3(42.7 \%)$ & 0.350 \\
\hline Grade 2 & 0 & 0 & \\
\hline Grade 3 & $5(35.7 \%)$ & $4(57.1 \%)$ & \\
\hline Grade 4 & 0 & 0 & \\
\hline Fortieth week (total) & $1(1.4 \%)$ & $3(3.6 \%)$ & 0.310 \\
\hline Grade 1 & $1(100 \%)$ & $3(100 \%)$ & \\
\hline Grade 2 & 0 & 0 & \\
\hline Grade 3 & 0 & 0 & \\
\hline Grade 4 & 0 & 0 & \\
\hline Periventricular leukomalacia & $2(2.7 \%)$ & $12(14.5 \%)$ & 0.009 \\
\hline Third day & 0 & 0 & \\
\hline Seventh day & $1(1.3 \%)$ & $3(3.8 \%)$ & 0.337 \\
\hline Fifteenth day & $2(2.9 \%)$ & $5(7.3 \%)$ & 0.237 \\
\hline Fortieth week & $1(1 . \%)$ & $7(10.0 \%)$ & 0.029 \\
\hline Basal ganglia lesions & $26(34.7 \%)$ & $51(61.5 \%)$ & 0.001 \\
\hline Third day & $21(28.0 \%)$ & $37(44.6 \%)$ & 0.031 \\
\hline Seventh day & $9(12.0 \%)$ & $34(43.0 \%)$ & $<0.001$ \\
\hline Fifteenth day & $3(4.3 \%)$ & $15(21.7 \%)$ & 0.002 \\
\hline Fortieth week & $1(1.4 \%)$ & $10(14.3 \%)$ & 0.005 \\
\hline Transient periventricular echodensities & $1(1.3 \%)$ & $5(5.02 \%)$ & 0.123 \\
\hline Patent ductus arteriosus & $33(44.0 \%)$ & $35(50.0 \%)$ & 0.469 \\
\hline Retinopathy of prematurity & $21(30.0 \%)$ & $22(31.4 \%)$ & 0.855 \\
\hline Any CUA & $30(40.0 \%)$ & $55(66.3 \%)$ & 0.001 \\
\hline Late CUAs & $1(1.4 \%)$ & $10(14.3 \%)$ & 0.005 \\
\hline Neonatal mortality & $6(8.0 \%)$ & $28(33.7 \%)$ & $<0.001$ \\
\hline
\end{tabular}

CUA, cranial ultrasound abnormality; IUGR, intrauterine growth restriction.

Considering that some neonates may not have IVH during the first days after birth, but after admission to the neonatal intensive care unit following postbirth events, such as respiratory problems and respiratory distress syndrome in particular, have been recognized as important risk factors in the development of germinal matrix hemorrhage-IVH $[22,23]$, we wanted to examine the course of IVH in these infants over time, similar to Cruz-Martinez et al. [13].
According to this study, the Doppler parameters including posterior cerebral artery peak systolic velocity, posterior cerebral artery PI, and posterior cerebral artery RI were negatively associated with the risk of IVH, PVL, BGLs, and any CUA. With an increase in the value of these Doppler parameters, the risk of neurological abnormalities decreased. While the associations between posterior cerebral artery peak systolic velocity and PVL and the posterior cerebral artery RI and IVH, PVL, BGLs, and 
Fig. 1. Frequency of intraventricular hemorrhage (IVH), periventricular leukomalacia (PVL), basal ganglia lesions (BGLs), any cranial ultrasound abnormality (CUA), and late CUAs among intrauterine growth restriction and control fetuses according to ductus venosus (a) and diastolic flow of umbilical artery status (b).
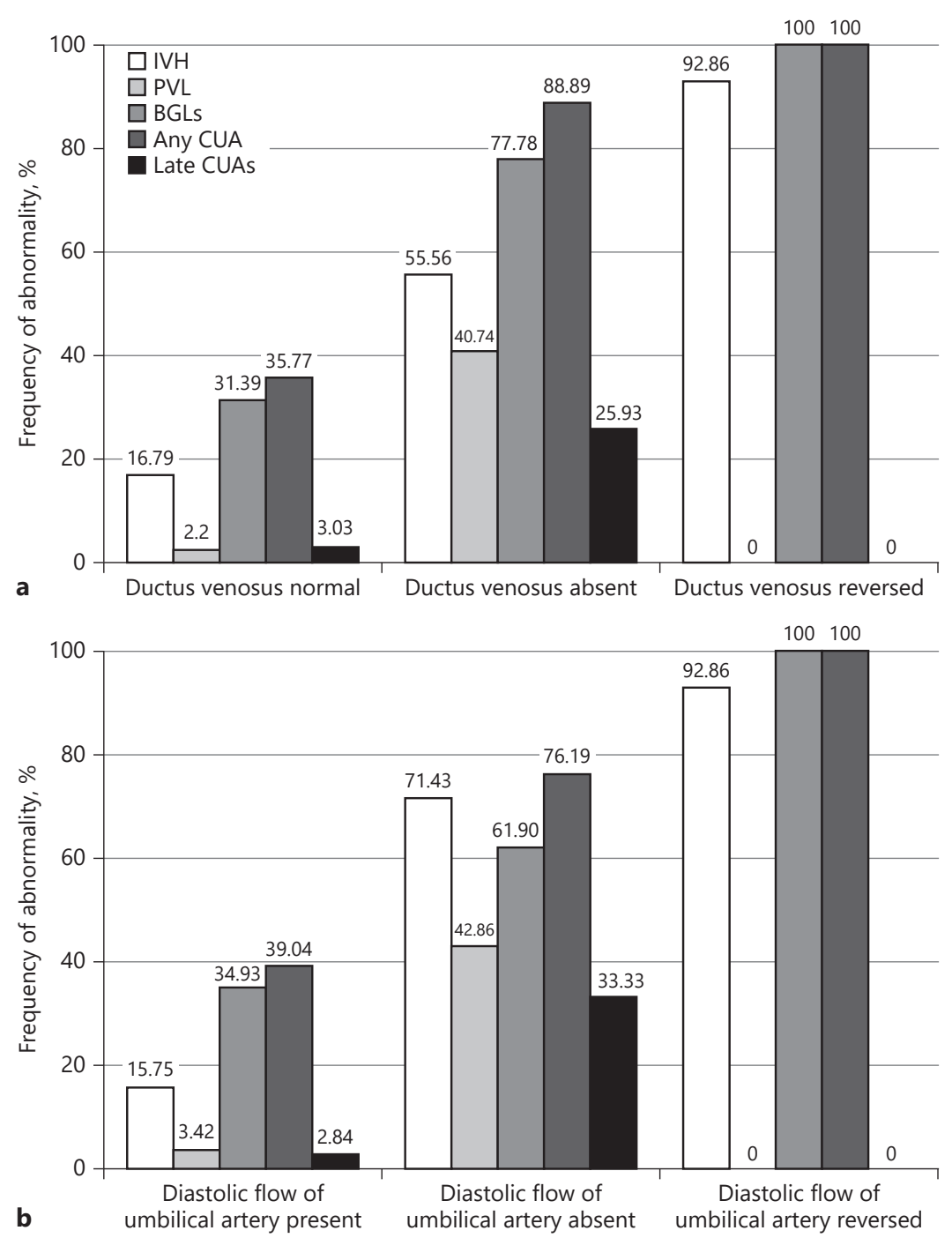

any CUA were protective, they were not statistically significant. A reason for such nonsignificant associations may be low sample size and insufficient power for detecting these associations. However, the clinical values of the reported ORs were important.

Prematurity may be a risk factor for abnormal development of the basal ganglia and thalami (BGT) [24]. Echogenicity of the BGT region (caudate nucleus, putamen, globus pallidus) is frequently seen on cranial US images obtained in preterm neonates $[25,26]$. In near-term neonates, increased echogenicity in this region usually indicates hypoxic-ischemic injury and is associated with serious neurological sequelae; however, the origin of echogenicity of the BGT in preterm infants is largely unclear [27].

Predictors of Cranial US Abnormalities in IUGR Fetuses
Echogenicity of the BGT was considered present if at least one cranial US examination revealed echogenicity of the BGT or areas within the BGT were increased compared with echogenicity of surrounding white matter on both coronal and sagittal images. Echogenicity of the BGT was described as diffuse or focal, homogeneous or inhomogeneous, and unilateral or bilateral. We recorded whether echogenicity was seen in the basal ganglia, thalami, or both. Lenticulostriate vasculopathy, seen as a punctate or linear echogenic structure in the distribution of thalamostriate vessels, was not considered echogenicity of the BGT. In another study, in 120 of 130 (92\%) preterm neonates echogenicity of the BGT was seen on sequential cranial US images obtained on admission. This was bilateral and diffuse in all 120 neonates. There was no 
Table 4. Associations of IUGR, GA, and Doppler parameters with IVH, PVL, BGLs, and any CUA

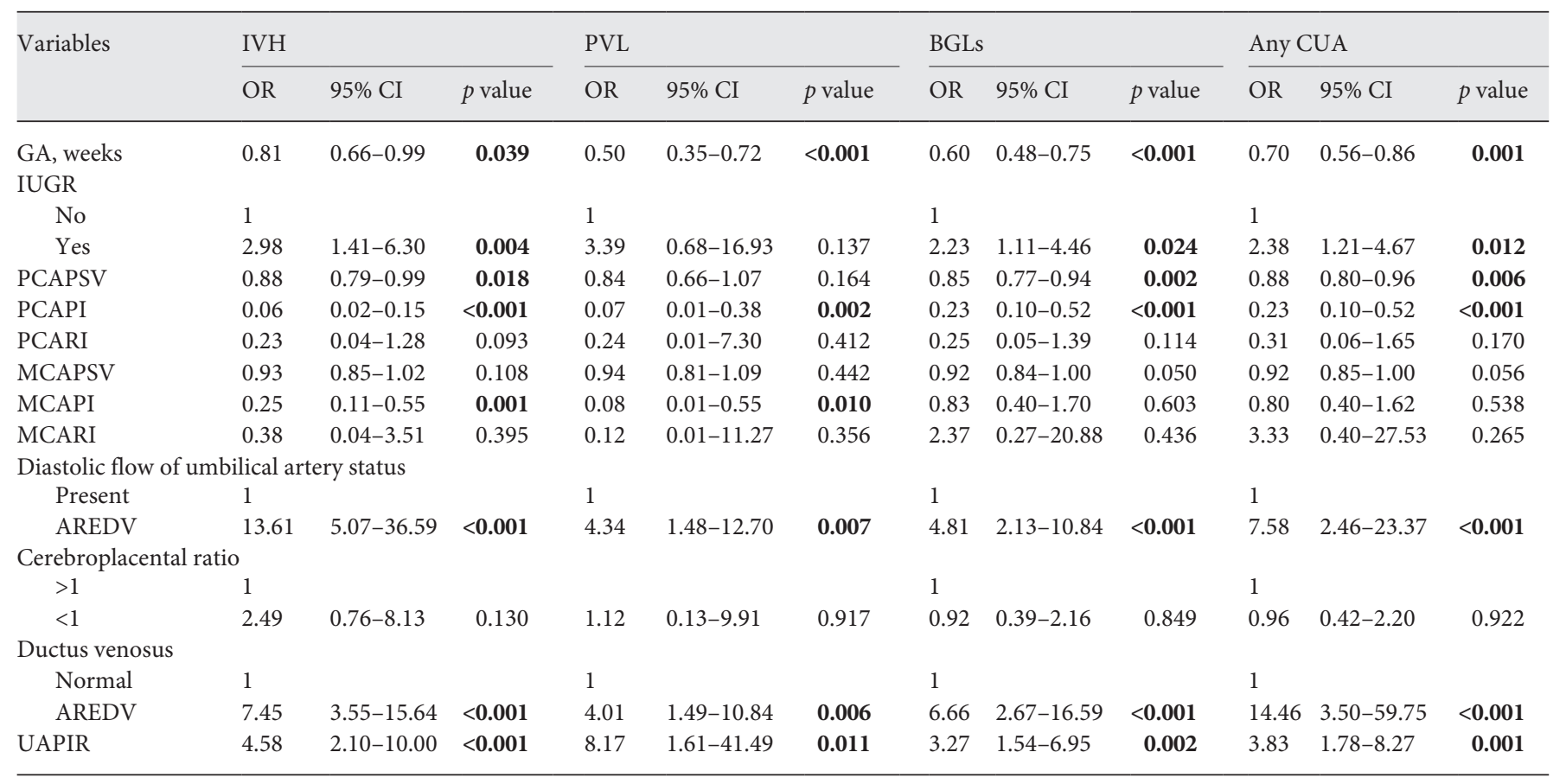

The associations of IUGR and all Doppler parameters were adjusted for GA. Bold values indicate statistical significance. AREDV, absent or reversed end-diastolic velocity; BGLs, basal ganglia lesions; CI, confidence interval; CUA, cranial ultrasound abnormality; GA, gestational age; IUGR, intrauterine growth restriction; IVH, intraventricular hemorrhage; MCAPI, middle cerebral artery pulsatility index; MCAPSV, middle cerebral artery peak systolic velocity; MCARI, middle cerebral artery resistance index; OR, odds ratio; PCAPI, posterior cerebral artery pulsatility index; PCAPSV, posterior cerebral artery peak systolic velocity; PCARI, posterior cerebral artery resistance index; PVL, periventricular leukomalacia; UAPIR, uterine artery pulsatility index right.

significant association between echogenicity of the BGT and nonphysiologic echodensities in the periventricular white matter [28].

In the review of texts it is stated that PVL is the major form of brain white matter injury affecting premature infants and that it is associated with subsequent development of cerebral palsy, intellectual impairment, and visual disturbances. The great risk of developing PVL is under 32 weeks of gestation. The incidence of PVL varies among centers and in relation with the imaging testing realized. Based on US, the frequency of PVL ranges from 5 to $15 \%$ in very-low-birth-weight infants [29]. This is in consistent with our study results.

PVL is detected in newborns by brain imaging using US, computed tomography, or magnetic resonance imaging. US is the initial standard method because it is portable and less expensive. The criteria for US diagnosis are not well defined. A standard examination includes coronal and sagittal view [30]. The US findings evolve on repeated examinations. Cysts appear after 1 or 3 weeks and disappear after 1 or 3 months if they are moderate or se- vere, ventriculomegaly may result [31]. The routine US recommended by the Quality Standard Subcommittee of the America Academy of Neurology and the Practice Committee of the Child Neurology Society made the following recommendations: Routine US screening should be performed on all infants with a GA $<30$ weeks. Screening should be performed at 7-14 days of age and repeated at 36-40 weeks postmenstrual age. This strategy is designed to detect unsuspected periventricular hemorrhagic infarction, development of posthemorrhagic hydrocephalus, or ventriculomegaly [29].

Another study by Padilla-Gomes et al. [5] compared the frequency of TPE, PVL, as well as lesions in fetal growth restriction preterm babies and in appropriate for gestational age babies. The GA of the study population ranged from 24 to 34 weeks, and brain changes were assessed by US at 3 days after delivery (US-I), 2 weeks after delivery (US-II), and term-equivalent age (US-III). The authors found a higher prevalence of TPE at US-I and PVL at US-II and US-III in fetal growth restriction neonates. Based on the results, the investigators proposed 
that fetal growth restriction is associated with an increased prevalence of white matter damage in US brain scans of preterm babies.

Increase in middle cerebral artery PI was significantly associated with a lower risk of IVH and PVL; however, its association with BGLs and any CUA was not statistically significant. The associations of middle cerebral artery peak systolic velocity and middle cerebral artery RI with neurological abnormalities were not statistically significant. The findings of this study suggest that the posterior cerebral artery and middle cerebral artery indices could be useful parameters for predicting the risk of neurological abnormalities. However, to determine the definite role of the above-mentioned Doppler parameters for managing fetuses, especially fetuses with IUGR, more studies with a larger sample size are suggested. Meher et al. [32], in a systematic review, stated that there was no increase in the risk of IVH with cerebral redistribution, regardless of GA, although one study observed an association with periventricular echodensities. These findings need to be interpreted with caution as they are based on observational data from a small number of studies of variable quality.

According to current evidence, the use of Doppler US examination among high-risk pregnant women can decrease the risk of neonatal mortality [33], but no sufficient evidence for all types of Doppler parameters is available. Among low-risk pregnant women, no definite evidence is available to show the advantages of the use of UA Doppler US or the combination of uterine and UA Doppler US for the mother or the fetus [34]. This study showed that AREDV in UA was a strong predictor of neonatal neurological abnormalities. The strongest association of this parameter was for IVH (OR = 13.61, 95\% CI 5.07-36.59). Regarding the associations between AREDV in UA and IVH, PVL, BGLs, and any CUA, they were completely in line with the results of a previous study, which reported ORs for IVH, PVL, BGLs, and any CUA as 4.94 (95\% CI 1.89-12.94), 4.07 (95\% CI 1.41-11.77), 9.91 (95\% CI 1.94-50.56), and 6.84 (95\% CI 2.77-16.92), respectively [16]. In the review of texts, it is stated that absent or reversed flow in the UA appears after $>50 \%$ of placental vessels have been obliterated [10]. Rekha et al. [35] concluded that careful Doppler evaluation can identify fetuses at risk for poor neonatal outcome in terms of neonatal intensive care unit admission and morbidity, thereby allowing antenatal risk estimation and prognostication. Another study stated that babies with abnormal UA Doppler studies were more than twice as likely to be admitted to the neonatal intensive care unit and to spend more time there [36]. Also the incidence of neurological

Predictors of Cranial US Abnormalities in IUGR Fetuses deficit was noticed at 6 months of follow-up in two babies (14.3\%) with severely abnormal UA velocimetry with absent diastole/reversed diastolic flow. The incidence of neurological deficit reported by Valcamonico et al. [37] was $36 \%$. In addition, the results of the current study were in line with those of other studies $[35,36,38,39]$.

In this study, AREDV in the DV was another strong predictor of all neurological outcomes including IVH, PVL, BGLs, and any CUA. This finding was in line with that of an earlier study [13]. In addition, this parameter was also recognized as a predictor of neonatal death [21, 40]. In the clinical view, prediction of the risk of neurological abnormalities is crucial and a major challenge for to the appropriate management of high-risk pregnancies. Overall, according to the results of this study and earlier studies [13, 38, 39], Doppler US parameters might be useful indices to predict the risk of neurological abnormalities and a better management of high-risk pregnancies.

The strength of this study was its prospective design and the accurate measurement of Doppler parameters, which can decrease the risk of information bias. A limitation of this study was its low sample size, which hindered the use of an adjusted logistic regression model to adjust the effect of other potential confounding variables. In this study, only the effect of GA was adjusted. Due to the low sample size and a large number of independent factors, sparse and confounding variables might have affected the validity of the results in the adjusted models [41]. Another limitation was the high correlation between the posterior and middle cerebral artery indices. When the highly correlated variables are included in the regression model simultaneously, the results might be spurious [42]. Therefore, the mentioned Doppler parameters could not be incorporated into the model simultaneously to report the adjusted results for all mentioned parameters. While the results of this study confirmed those of earlier studies, prospective cohort studies with a larger sample size are required to confirm the results of this study. Also, the diagnosis of TPE and PVL grade I and II is highly subjective and subject to interobserver variability. Therefore, a better approximation to the existence or not of damage of the white matter would have been magnetic resonance imaging.

In conclusion, according to the results of this study, GA and AREDV in UA status and DV were reliable predictors of neurological abnormalities in fetuses. The risk of neurological abnormalities and neonatal death among fetuses with IUGR was considerable. The value of Doppler US parameters was shown as a predictor of neurological abnormalities among fetuses, especially fetuses with IUGR in pregnant women. 


\section{Acknowledgment}

The authors would like to thank the sincere and meticulous efforts of the patients and all staff of Vali Asr Hospital. The Vali Asr Research Center for Reproductive Health provided all of the financial support for the conduct of this study.

\section{Disclosure Statement}

The authors declare no conflict of interest.

\section{References}

1 Goldenberg RL, Cliver SP: Small for gestational age and intrauterine growth restriction: definitions and standards. Clin Obstet Gynecol 1997;40:704-714.

2 Arduini D, Rizzo G: Normal values of pulsatility index from fetal vessels: a cross-sectional study on 1556 healthy fetuses. J Perinat Med 1990;18:165-172.

3 Bernstein IM, Horbar JD, Badger GJ, Ohlsson A, Golan A: Morbidity and mortality among very-low-birth-weight neonates with intrauterine growth restriction. Am J Obstet Gynecol 2000;182:198-206.

4 Pisani F, Leali L, Moretti S, Turco E, Volante E, Bevilacqua G: Transient periventricular echodensities in preterms and neurodevelopmental outcome. J Child Neurol 2006;21:230235.

5 Padilla-Gomes NF, Enríquez G, Acosta-Rojas R, Perapoch J, Hernandez-Andrade E, Grata$\cos$ E: Prevalence of neonatal ultrasound brain lesions in premature infants with and without intrauterine growth restriction. Acta Paediatr 2007;96:1582-1587.

6 Allen MC: Neurodevelopmental outcomes of preterm infants. Curr Opin Neurol 2008;21: 123-128.

7 Omizzolo C, Scratch SE, Stargatt R, Kidokoro $\mathrm{H}$, Thompson DK, Lee KJ, et al: Neonatal brain abnormalities and memory and learning outcomes at 7 years in children born very preterm. Memory 2014;22:605-615.

8 van den Hout BM, Stiers P, Haers M, van der Schouw YT, Eken P, Vandenbussche E, et al: Relation between visual perceptual impairment and neonatal ultrasound diagnosis of haemorrhagic-ischaemic brain lesions in 5year-old children. Dev Med Child Neurol 2000;42:376-386.

9 Habek D, Jugović D, Hodek B, Herman R, Matičević A, Habek JČ, et al: Fetal biophysical profile and cerebro-umbilical ratio in assessment of brain damage in growth restricted fetuses. Eur J Obstet Gynecol Reprod Biol 2004; 114:29-34.

10 Figueras F, Gardosi J: Intrauterine growth restriction: new concepts in antenatal surveillance, diagnosis, and management. Am J Obstet Gynecol 2011;204:288-300.

11 Kiserud T, Kessler J, Ebbing C, Rasmussen S: Ductus venosus shunting in growth-restricted fetuses and the effect of umbilical circulatory compromise. Ultrasound Obstet Gynecol 2006;28:143-149.
12 Baschat A, Gembruch U, Viscardi R, Gortner L, Harman C: Antenatal prediction of intraventricular hemorrhage in fetal growth restriction: what is the role of Doppler? Ultrasound Obstet Gynecol 2002;19:334-339.

13 Cruz-Martinez R, Tenorio V, Padilla N, Crispi F, Figueras F, Gratacos E: Risk of ultrasound-detected neonatal brain abnormalities in intrauterine growth-restricted fetuses born between 28 and 34 weeks' gestation: relationship with gestational age at birth and fetal Doppler parameters. Ultrasound Obstet Gynecol 2015;46:452-459.

14 Figueras F, Meler E, Iraola A, Eixarch E, Coll $\mathrm{O}$, Figueras J, et al: Customized birthweight standards for a Spanish population. Eur J Obstet Gynecol Reprod Biol 2008;136:20-24.

15 de Vries LS, Eken P, Dubowitz LM: The spectrum of leukomalacia using cranial ultrasound. Behav Brain Res 1992;49:1-6.

16 van Wezel-Meijler G, van der Knaap MS, Sie LT, Oosting J, van Amerongen AH, Cranendonk A, et al: Magnetic resonance imaging of the brain in premature infants during the neonatal period. Normal phenomena and reflection of mild ultrasound abnormalities. Neuropediatrics 1998;29:89-96.

17 Volpe JJ: Intracranial hemorrhage: germinal matrix-intraventricular hemorrhage of the premature infant; in Volpe JJ (ed): Neurology of the Newborn. Philadelphia, Saunders, 2008, pp 517-588.

18 Ministry of Health and Medical Education: Country Guide for Midwifery and Women Services. Tehran, Family and Population Health Office, Mothers Health Office, 2017.

19 Gole GA, Ells AL, Katz X, Holmstrom G, Fielder AR, Capone A Jr, et al: The International Classification of Retinopathy of Prematurity revisited. Arch Ophthalmol 2005;123: 991-999.

20 Bone RC, Balk RA, Cerra FB, Dellinger RP, Fein AM, Knaus WA, et al: Definitions for sepsis and organ failure and guidelines for the use of innovative therapies in sepsis. Chest 1992;101:1644-1655.

21 Cruz-Lemini M, Crispi F, van Mieghem T, Pedraza D, Cruz-Martínez R, Acosta-Rojas R, et al: Risk of perinatal death in early-onset intrauterine growth restriction according to gestational age and cardiovascular Doppler indices: a multicenter study. Fetal Diagn Ther 2012;32:116-122.
22 Vela-Huerta MM, Amador-Licona M, Medina-Ovando N, Aldana-Valenzuela C: Factors associated with early severe intraventricular haemorrhage in very low birth weight infants. Neuropediatrics 2009;40:224-227.

23 Kaiser JR, Gauss CH, Pont MM, Williams DK: Hypercapnia during the first 3 days of life is associated with severe intraventricular hemorrhage in very low birth weight infants. J Perinatol 2006;26:279-285.

24 Inder TE, Warfield SK, Wang H, Huppi PS, Volpe JJ: Abnormal cerebral structure is present at term in premature infants. Pediatrics 2005; 115:286-294.

25 Leijser L, Klein R, Veen S, Liauw L, van Wezel-Meijler G: Hyperechogenicity of the thalamus and basal ganglia in very preterm infants: radiological findings and short-term neurological outcome. Neuropediatrics 2004; 35:283-289.

26 Soghier LM, Vega M, Aref K, Reinersman GT, Koenigsberg M, Kogan M, et al: Diffuse basal ganglia or thalamus hyperechogenicity in preterm infants. J Perinatol 2006;26:230-236.

27 Eken P, Jansen GH, Groenendaal F, Rademaker KJ, de Vries LS: Intracranial lesions in the fullterm infant with hypoxic ischaemic encephalopathy: ultrasound and autopsy correlation. Neuropediatrics 1994;25:301-307.

28 van Wezel-Meijler G, Leijser LM, Wiggers-de Bruïne FT, Steggerda SJ, van der Grond J, Walther FJ: Diffuse hyperechogenicity of basal ganglia and thalami in preterm neonates: a physiologic finding? Radiology 2011;258: 944-950.

29 Barría RM, Flández A: Parenchymatous brain injury in premature infants: intraventricular hemorrhage and periventricular leukomalacia; in: Neonatal Care. Chile, IntechOpen, 2012, pp 87-102. https://www.intechopen. com/books/neonatal-care/parenchymatousbrain-injury-in-premature-infants-intraventricular-hemorrhage-and-periventricularleu.

30 Veyrac C, Couture A, Saguintaah M, Baud C: Brain ultrasonography in the premature infant. Pediatr Radiol 2006;36:626-635.

31 Blankenberg FG, Loh NN, Norbash AM, Craychee JA, Spielman DM, Person BL, et al: Impaired cerebrovascular autoregulation after hypoxic-ischemic injury in extremely lowbirth-weight neonates: detection with power and pulsed wave Doppler US. Radiology 1997; 205:563-568 
32 Meher S, Hernandez-Andrade E, Basheer SN, Lees C: Impact of cerebral redistribution on neurodevelopmental outcome in small-forgestational-age or growth-restricted babies: a systematic review. Ultrasound Obstet Gynecol 2015;46:398-404.

33 Alfirevic Z, Stampalija T, Gyte GM: Fetal and umbilical Doppler ultrasound in high-risk pregnancies. Cochrane Database Syst Rev 2013;11:CD007529.

34 Alfirevic Z, Stampalija T, Medley N: Fetal and umbilical Doppler ultrasound in normal pregnancy. Cochrane Database Syst Rev 2015; 4:CD001450.

35 Rekha B, Pavanaganga A, Nagarathnamma R: Comparison of Doppler findings and neonatal outcome in fetal growth restriction. Int J Reprod Contracept Obstet Gynecol 2017;6: 955-958.
36 Devendra A, Desai S, Sheth P, Prema K: Significance of umbilical artery velocimetry in perinatal outcome of growth restricted foetuses. J Obstet Gynecol India 2005;55:138143.

37 Valcamonico A, Danti L, Frusca T, Soregaroli M, Zucca S, Abrami F, et al: Absent enddiastolic velocity in umbilical artery: risk of neonatal morbidity and brain damage. Am J Obstet Gynecol 1994;170:796-801.

38 Baschat A, Viscardi R, Hussey-Gardner B, Hashmi N, Harman C: Infant neurodevelopment following fetal growth restriction: relationship with antepartum surveillance parameters. Ultrasound Obstet Gynecol 2009; 33:44-50.
39 Shand AW, Hornbuckle J, Nathan E, Dickinson JE, French NP: Small for gestational age preterm infants and relationship of abnormal umbilical artery Doppler blood flow to perinatal mortality and neurodevelopmental outcomes. Aust NZ J Obstet Gynaecol 2009;49: 52-58.

40 Baschat AA, Cosmi E, Bilardo CM, Wolf $\mathrm{H}$, Berg C, Rigano S, et al: Predictors of neonatal outcome in early-onset placental dysfunction. Obstet Gynecol 2007;109(2 Pt 1):253-261.

41 Greenland S, Mansournia MA, Altman DG: Sparse data bias: a problem hiding in plain sight. BMJ 2016;352:i1981.

42 Steyerberg EW: Clinical Prediction Models: A Practical Approach to Development, Validation, and Updating. New York, Springer Science and Business Media, 2008. 